\title{
The Course of Spindle Formation in the Spermatocyte of the Grasshopper, Acrydium japonicus, Observed by Phase Microscopy
}

Ken-ya Kawamura

Biological Department, Tokyo Metropolitan University, Tokyo

Recsived June 30,1954

Since Bèlary's publication (1929) concerning the mitosis in the grasshopper Chorthippus, many investigations have been carried out. But the studies appeared after Bĕlař were performed with fixed and stained materials. Much has remained unknown on mitotic apparatus and events in the living cell. Recently Shimakura $(1952,1953)$ has reported detailed observations on the spindle formation in the living grasshopper spermatocytes.

By light microscopy, the detection of the spindle body in the living cell is very difficult. However, by phase microscope, by the aid of both the arrangement of the mitochondria and a slight difference in the refractive index between the spindle body substance and its surrounding cytoplasm, it is possible to trace the course of the spindle formation. ${ }^{\text {. }}$

\section{Method}

The present study was performed with male germ cells of the grasshopper, Acrydium japonicus, abundant during summer in the neighborhood of Tokyo. All observations were based on the primary spermatocytes derived from nymphal specimens.

In order to trace the course of the spindle formation in the living spermatocyte, the hanging-drop method with the addition of the body fluid was exclusively used. By carefully cutting the follicle walls with a sharp knife, germ cells with a small amount of body fluid are taken out onto the cover slip which was then inverted over a depression slide, and sealed with liquid paraffin. Above mentioned procedure requires sufficient care and quickness.

The observations were performed with the aid of a phase contrast microscope, using Olympus light sourse (6V. 5A), Zettnow's filter solution, periplanocular $\times 10$ (Olympus), and bright medium contrast optics $\times 90$ (Tiyoda), at temperature of about $23^{\circ} \mathrm{C}$.

The photomictographs were taken with a PM-6 (Olympus) by using Eastman Kodak $35 \mathrm{~mm}$ Plus X film.

\section{Observations}

The course of the intra-cellular changes was traced successively in a

1 The author is under deep obligation to Professor Katsuma Dan for his kind guidance and kind aid in the preparation of this manuscript. 
single cell from diakinesis till metaphase, with special reference to the formation of the spindle body (Figs. 1,2 and 5-8).

At the late diakinesis, the spindle-like-body (cytoplasmic spindle) with the length of about $1 / 2$ cell diameter was formed in the cytoplasm (Fig. 1). The mitochondria were granular at the outset, but they became thready with the laps of time, and these mitochondria were arranged in the spindle shape.
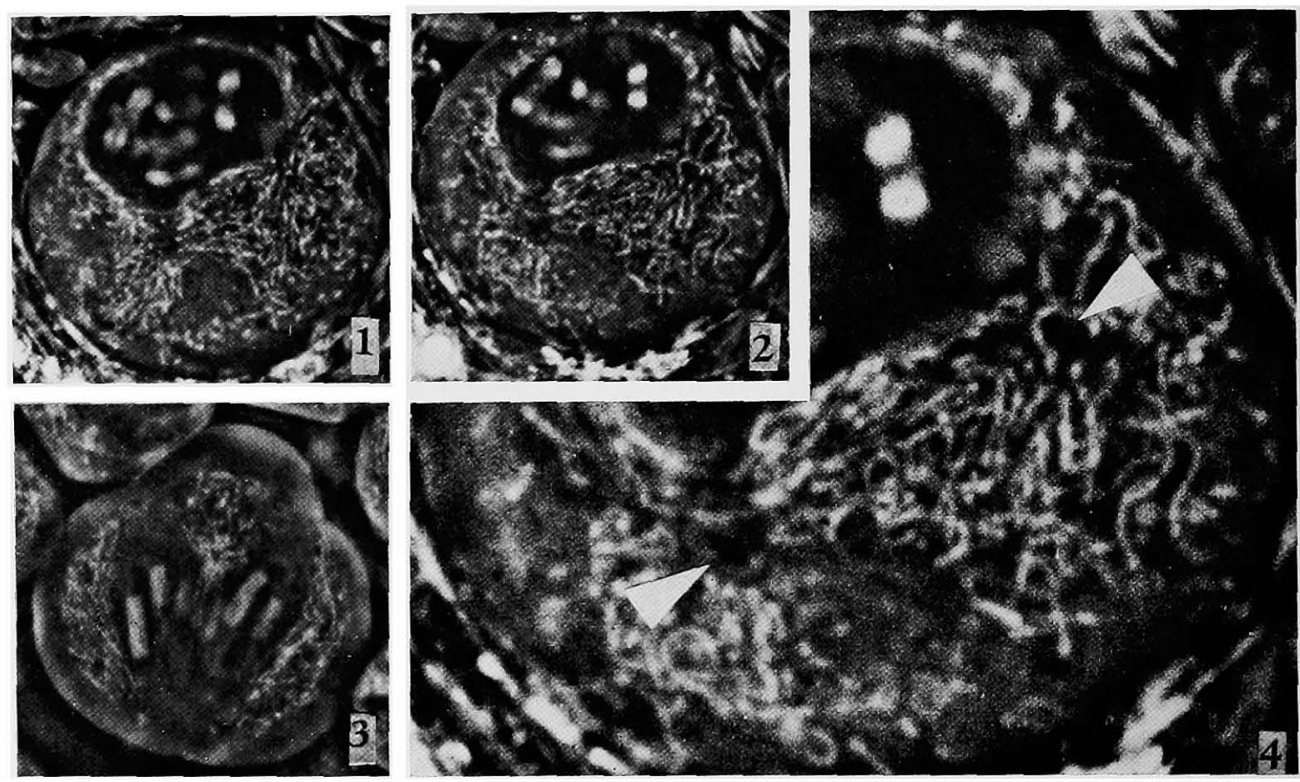

Figs. 1-4. All figures are of the primary spermatocytes of Acrydium japonicus. 1-3, $\times 1000.4, \times 2000.1,2$ and 4 , late diakinesis-cells showing the formation of the cytoplasmic spindle. Fig. 4 is the enlarging of Fig. 2, showing two centrioles per each pole of the cytoplasmic spindle. 3 , a hydrated binucleate cell at metaphase.

Two centrioles per each pole of the cytoplasmic spindle were detected as two dark points by the bright contrast optics (Figs. 2 and 4). The poles gradually separated away from each other, and attached themselves to the cell surface layer. At this time, the intact nuclear membrane near the cytoplasmic spindle and the chromosomes contained within it were gently drawn to each pole. The distinctness of the nuclear membrane, thereafter, was lost from the area near the cytoplasmic spindle. The mitochondria on the cytoplasmic spindle were pushed ahead by the nuclear contents which fills the inside of the cytoplas mic spindle and makes it thicker. The definitive spindle was thus formed.

The mitochondria thus pushed aside by the nuclear contents were distributing in a uniform density arround the spindle body.

Finally the chromosomes were gathered to the equatorial plate, and the cell entered into the metaphase.

During all these processes, the arrangement of the mitochondria was kept along the spindle contour. 
Sometimes, with changing temperature, the water evaporated from the hanging-drop or condensed on it which, in turn, led to dehydration or hydration of the cells. Figure 3 shows a case of hydration of a binucleate cell at metaphase; the depressions were formed on the polar parts of the spindle bodies, which might suggest that the spindle poles are anchored to the cell surface layer by means of their accompanying centrioles.
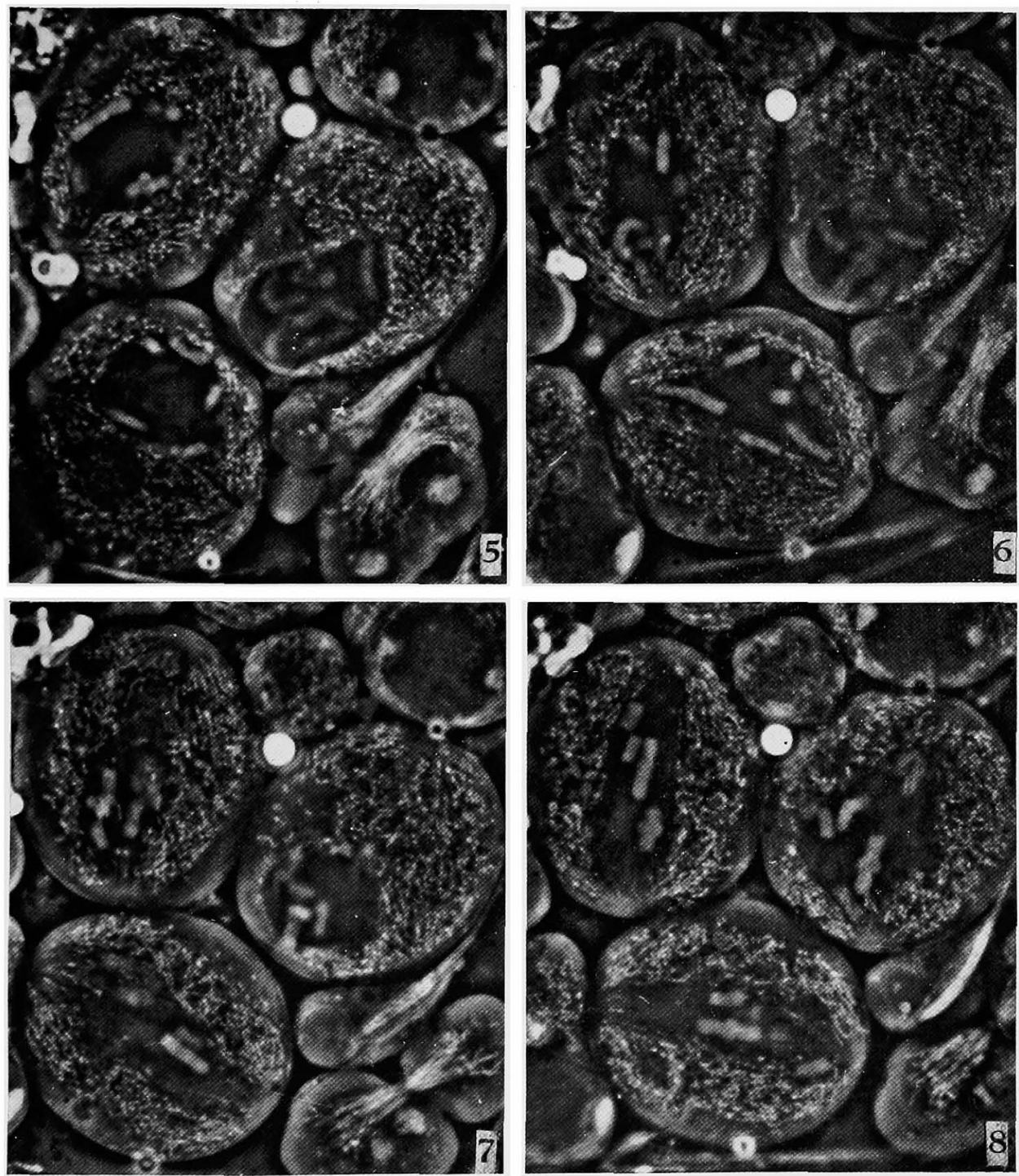

Figs. 5-8. Photomicrographs of the primary spermatocytes of Acrydium japonicus taken with the aid of the phase microscope. Successive series of the intra-cellular changes during from diakinesis to metaphase. $\times 1000$.

\section{Remarks}

Although there have been debates upon the reality of the spindle body for a long time, this question seems to have been settled by the isolation of 
the mitotic apparatus in the sea urchin egg by Mazia and Dan (1952) and also by the polarization microscopic demonstration of the spindle body in living cell by Inoue (1953). These studies were, however, mostly done with the stages of from metaphase to telophase.

The arrangement of the mitochondria in the shape of the spindle body was shown in Figure 1. The author supposes the existence of some basic structure in the cytoplasm to make such mitochondrial arrangement possible, to which the nuclear contents are later added to complete the spindle.

The detailed observations on the formation of the spindle body in the spermatocytes of grasshopper (Chrysochraon japonicus) have been reported by Shimakura $(1952,1953)$ in which he thinks that the spindle-like-body which is formed in the cell before the breakdown of the nuclear membrane is the simple connection of the astral rays from the opposite poles, and the real spindle of the first division is formed from the nuclear contents at the late prophase.

In the final analysis, the results in the present study were much the same as Shimakura's.

Moreover, there are certain divisions in the salamander (Meves 1887), as well as in the egg of the mollusc Arion (Lams 1910), where continuous fibers arise between two centers early in the mitotic cycle and form a miniature spindle. And this quite definitely occurs outside of the nucleus, and such a spindle gradually assumes metaphase proportion before the nuclear membrane breaks down.

On the contrary, Wada (1950) recently proposed that the spindle body in the plant cell (Tradescantia) was derived solely from the nuclear contents, but there will be difficulty to extend this idea to animal mitoses generally.

Thereupon, if the conjecture about the origin of the spindle body is allowed, the spindle body may be said to have its precursor in the cytoplasm.

When the metaphase cell was put into a hypotonic medium, two suggestions will be given from its appearance: The poles of the spindle body may be anchored to the cell surface layer, and the spindle body may be a considerably hard structure. Bělař (1929) has reported that in the hypertonic media, there is shrinkage of the spindle body, but this shrinkage is much greater in the transverse than in the longitudinal direction. This result will be expected from the above notion.

\section{Summary}

The course of the intra-cellular changes in a single spermatocyte of Acrydium japonicus was observed successively from diakinesis to metaphase in the living condition with the aid of the phase microscopy.

Cytoplasmic precursor of the spindle body, the anchorage of the spindle poles to the cell surface layer and the rigidity of the spindle body were discussed. 


\section{Literature cited}

Bělař, K. 1929. Beiträge zur Kausalanalyse der Mitose. II. Untersuchungen an den Spermatozyten von Chorthippus (Stenobothrus) lineatus Panz. Roux' Arch. Entw. 118: 359-484.

Inoué, S. 1953. Polarization optical studies of the mitotic spindle. I. The demonstration of spindle fibers in living cells. Chromosoma 5: 487-500.

Lams, H. 1910. Recherches sur l'oeluf d'Arion empiricorum. Acad. roy. Belg., Classe de Sciences 2: 1-170.

Mazia, D. and K. Dan 1952. The isolation and biochemical characterization of the mitotic apparatus of dividing cells. Proc. Nat. Acad. Sci. 38: 826-838.

Moves, F. 1897. Über die Entwickelung der männlchen Geschlechtszellen von Salamandra maculosa. Arch. mikr. Anat. 48: 1-83,

Shimakura, K. 1952. Behavior of mitochondria in dividing spermatocyte of grasshopper I. (in Japanese) The Dobutsugaku Zasshi 61: 100.

- 1953. Behavior of mitochondria in dividing spermatocyte of grasshopper II. (in Japanese) The Dobutsugaku Zasshi 62: 91.

Wada, B. 1950. The mechanism of mitosis based on studies of the submicroscopic structure and of the living state of the Tradescantia cell. Cytologia 16: 1-26. 